\title{
DEVELOPMENT OF SPECIAL MEASURING TOOL FOR TRACE OF MICRO FORCE VALUE
}

\author{
Li Yang ${ }^{1}$, Shi Wei ${ }^{2}$, Tao Jizeng ${ }^{3}$ \\ ${ }^{1}$ Changcheng Insitiute of Metrology \& Measurement, Beijing, China, 1y304cimm@126.com \\ ${ }^{2}$ Changcheng Insitiute of Metrology \& Measurement, Beijing, China, swlltm@126.com \\ ${ }^{3}$ Changcheng Insitiute of Metrology \& Measurement, Beijing, China, tjz 75@163.com
}

\begin{abstract}
:
This paper introduces the development of the special traceability measuring tool for micro force value with the test force range of $0.009807 \mathrm{~N}$ $9.807 \mathrm{~N}(1 \mathrm{gf} \sim 1 \mathrm{kgf})$ and the accuracy of better than $\pm 0.1 \%$, including the selection of the electromagnetic balance module, the design of the hardware structure, and the compilation of the software program. Finally, the special traceability measuring tool integrating the test measurement, automatic data collection and automatic data processing is realized, and the test verification result is given - It provides a method to solve the problem of trace the small force value of existing micro Vickers hardness tester and Knoop hardness tester.
\end{abstract}

Keywords: micro force, measuring tool, traceability, micro Vickers hardness, Knoop hardness

\section{INTRODUCTION}

With the development of modern material surface engineering, such as Physical vapor deposition, magnetron sputtering, ion implantation, surface modification, thermal spraying, nitriding and carburizing, etc., the thickness of micro Vickers hardness and Knoop hardness test samples or the surface modified layer on the sample surface is getting thinner and thinner, and the hardness test force value is getting lower and lower, reaching $0.009807 \mathrm{~N}$. However, the minimum measuring range of the existing force measuring instrument is $1 \mathrm{~N}$. According to the principle of one tenth use, the minimum measurement can be $0.1 \mathrm{~N}$, which can not meet the existing requirements. Therefore, it is very necessary and urgent to develop the special traceability measuring tool with the lower limit of $0.009807 \mathrm{~N}$.

\section{PROJECT DESIGN}

In this paper, the special traceability measuring tool for micro force value is developed, which has the force value range of $0.009807 \mathrm{~N} \sim 9.807 \mathrm{~N}(1$ gf $\sim 1 \mathrm{kgf}$ ) and the accuracy of better than $\pm 0.1 \%$. It selects the electromagnetic balance module as the core, and carries on the package design to it, has realized the test measurement, the data automatic collection, the data automatic processing as a whole special traceability measurement tool, specifically includes, the electromagnetic balance module selection design, the hardware structure design, and the software programming three parts.

The output value of the balance is the mass. The relationship between the force value and the mass is shown in formula (1).

$F=m g\left(1-\frac{\rho_{1}}{\rho_{2}}\right)$

In the formula:

$F$ - force value, $\mathrm{N}$;

$m$ - mass, $\mathrm{kg}$;

$g$ - the local acceleration of gravity in the laboratory, $\mathrm{m} / \mathrm{s}^{2}$;

$\rho_{1}$-air density, $\mathrm{kg} / \mathrm{m}^{3}$;

$\rho_{2}$-weight density, $\mathrm{kg} / \mathrm{m}^{3}$.

Since the density of the weight is far greater than that of the air, $\frac{\rho_{1}}{\rho_{2}}$ is negligible.

\subsection{Selection and design of electromagnetic balance module}

According to the research, referring to the test requirements, working space of most hardness tester and other factors, by selecting the range, accuracy, geometric dimension and other indicators of electromagnetic balance module, the two electromagnetic balance modules of sartorius are finally selected, their models are WZA224-N and WZA1203-N, as shown in Figure 1, and their technical indicators are shown in Table 1.

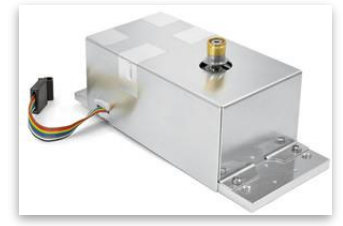

a) WZA224-N

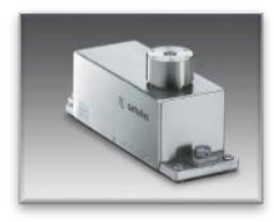

b) WZA1203-N
Figure 1 electromagnetic balance modules 
Table 1 Technical indicators of lectromagnetic balance modules

\begin{tabular}{|c|c|c|}
\hline Model & WZA224-N & WZA1203-N \\
\hline $\begin{array}{c}\text { Weighing } \\
\text { Capacity(g) }\end{array}$ & 220 & 1200 \\
\hline Readability(mg) & 0.1 & 1 \\
\hline $\begin{array}{c}\text { Maximum } \\
\text { deviation(mg) }\end{array}$ & \pm 1 & \pm 5 \\
\hline $\begin{array}{c}\text { Standard deviation } \\
\text { determined(mg) }\end{array}$ & 0.1 & \pm 2 \\
\hline $\begin{array}{c}\text { Maximum } \\
\text { Linearity(mg) }\end{array}$ & \pm 0.2 & $200 \times 67 \times 78$ \\
\hline $\begin{array}{c}\text { Physical } \\
\text { dimension(mm) }\end{array}$ & $181 \times 67 \times 73$ & 1 \\
\hline
\end{tabular}

Although the electromagnetic balance module with the smallest geometric size is selected on the premise of meeting the requirements of technical specifications, its length and width meet the requirements of micro Vickers hardness tester in our laboratory. Because the size of the micro Vickers hardness tester in our laboratory is $200 \mathrm{~mm} \times 280$ $\mathrm{mm}$. But its height is still higher than the working space height of most hardness machines, including those in our laboratory. Therefore, it is necessary to reform the selected electromagnetic balance module, remove its own measuring head and replace it with a thinner force measuring base. Because the selected balance module is a mature commodity, it has a certain stiffness, and because the indenters used in micro Vickers hardness test and Knoop hardness test are diamond indenters, it is necessary to set a ceramic force measuring unit in the center of the force measuring seat to ensure its stiffness in use and reduce the impact on the test results. The designed balance module has good stiffness, which can reduce the influence of the deformation of the worktable on it in the use process. The accuracy of the design and operation is $\pm 0.01 \%$, and the influence of the table deformation can be ignoredThe design drawing of electromagnetic balance module transformation is shown in Figure 2.

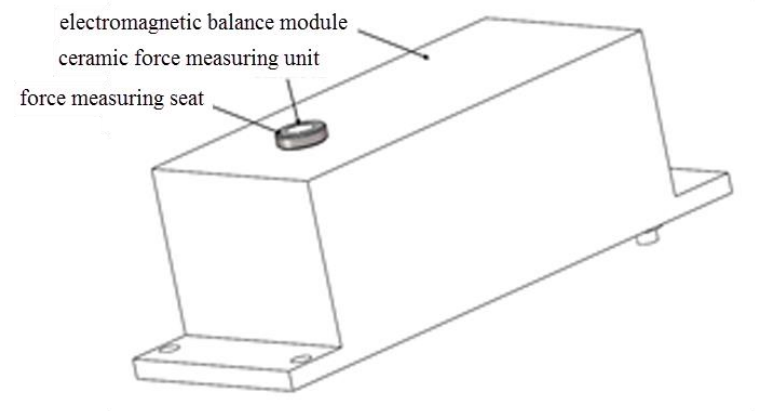

Figure 2 Design drawing of electromagnetic balance module transformation

\subsection{Hardware structure design}

The special measuring tool for trace of micro force value is often carried out, so it needs to be easy to carry, simple to make mistakes, and anti-collision and other functions. Therefore, hardware structure design should be carried out on the basis of electromagnetic force balance module. The hardware structure design diagram is shown in Figure 3.

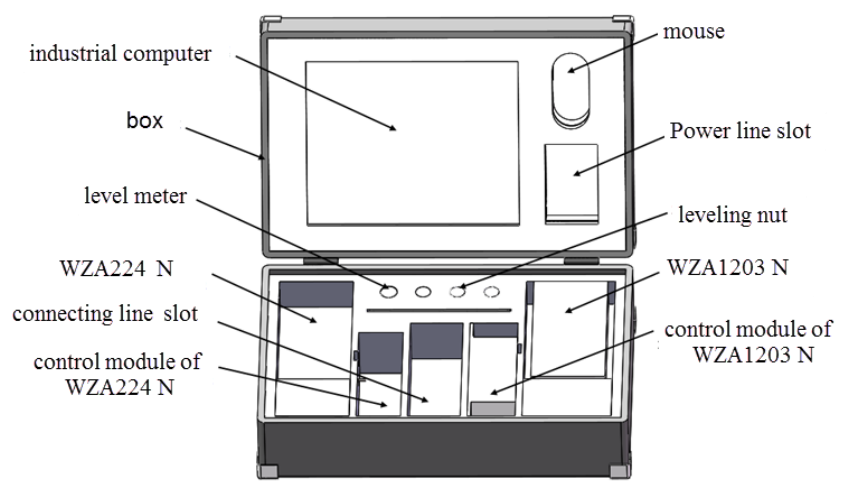

Figure 3 Hardware structure design drawing

After completion, the special measuring tools for trace the micro force value include the box with pull rod, industrial computer, mouse, power supply, two modified electromagnetic balance modules and their corresponding control board, level meter, leveling nut, etc.

The box with pull rod is made of aluminum alloy with light weight, high strength and easy to carry and move. The box is filled with thick sponge to protect internal parts. The industrial computer is used for operation test, collection and processing of test data. The level meter and leveling nut are used to ensure the level of the device during the test. The WZA224-N module and WZA1203-N module are used for test force The control module is used to control the electromagnetic balance module, and the trunking and mouse are used to link and operate.

\subsection{Programming and design of software}

In order to reduce the error of manual calculation, the special traceability measurement tool for micro force value is designed and compiled with supporting automatic processing software, which adopts automatic reading, automatic calculation and other functions.

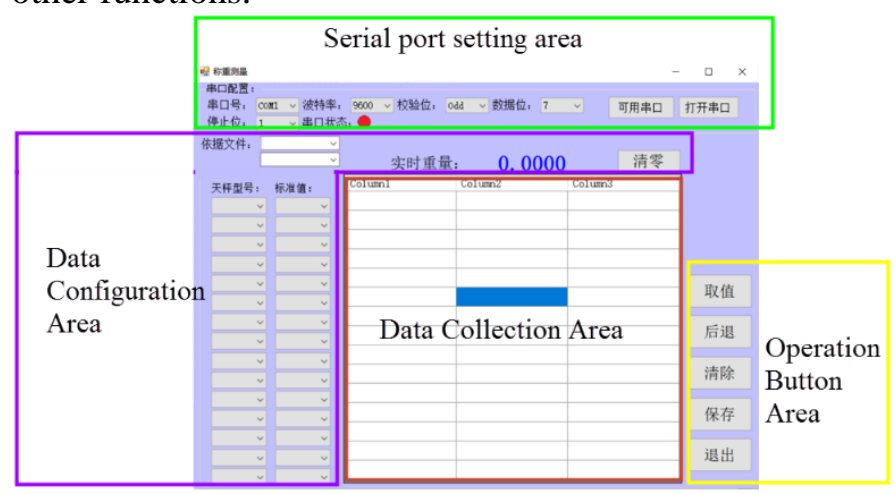

Figure 4 Automatic processing software interface 
As shown in Figure 4, the automatic processing software is divided into four areas, namely:

\section{a) Serial port setting area}

The serial port can be set in this area for communication with the electromagnetic balance module.

It is written in C\# language, using standard serial port communication protocol and SBI communication protocol based on te series electronic balance module. The communication steps are as follows:

Create serial port: serialport SP1 = new serialport();

Open the serial port: Sp1. Open();

Read serial data: Sp1. Readline();

Close the serial port: Sp1. Close();

As shown in Figure 5, analyze the data according to the SBI protocol of the electromagnetic balance module, take the first to the 14th bit of data, remove the useless space, and display the data on the interface.

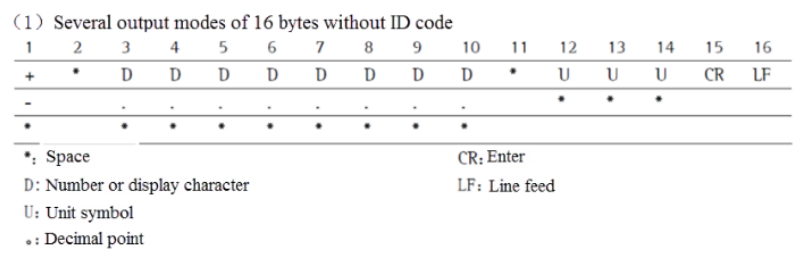

Figure 5 SBI communication protocol of electromagnetic balance module

b ) Data configuration area

In this area, we can select the reference standards, model and measurement standard value, and have the function of clearing before the test.

Reference standards include ISO 6507 Metallic materials - Vickers hardness test, ASTM E 92 Standard test methods for Vickers hardness and Knoop hardness of metallic materials, ASTM E 384 Standard test method for microindentation hardness of materials, and JJG 148 Verification regulation of Vickers hardness reference blocks, and JJG 151 Verification regulation of metallic Vickers hardness testers.

Refer to the protocol of electromagnetic balance module, as shown in Figure 6, set the equipment reset function, and the command format is as follows:

sp1.WriteLine("ESC\rV\r\n");

\begin{tabular}{ll}
\hline$T$ & Tare and zero \\
\hline$U$ & Tare \\
\hline $\mathrm{V}$ & Zero \\
\hline $\mathrm{W}$ & External adjustment / calibration \\
\hline
\end{tabular}

Figure 6 protocol of electromagnetic balance module

c) Data collection area

This area is used to display the collected and processed data.

\section{d) Operation button area}

Buttons in this area are used for software operation, including value taking, backward, clear, save and exit.

After data acquisition, when the "value taking" button is pressed, the software will intercept the current test value and save it to the data acquisition area. The backward button deletes the last data in the acquisition area. The clear button clears all data in the data collection area. The save button saves the data in the data collection area to the record report. The exit button will push out of the system

Physical drawing of development of the special traceability measuring tool for micro force value, as shown in Figure 7.

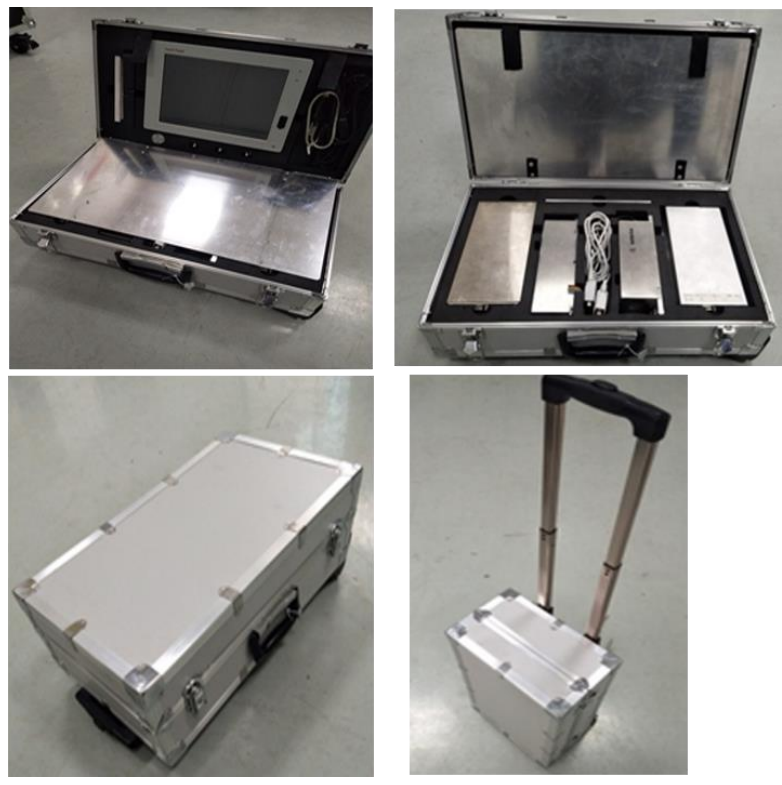

Figure 7 Physical drawing of development of the special traceability measuring tool for micro force value

\subsection{Test verification}

In order to verify whether the special measuring tool for tracing of micro force value meets the required technical indicators, the test is carried out. See Table 2 for the standard measuring instruments, and see Table 3 for test results.

Table 2 Standard measuring instruments

\begin{tabular}{|c|c|c|c|}
\hline Name & Model & Accuracy level & Number \\
\hline $\begin{array}{c}\text { Standard } \\
\text { weights }\end{array}$ & $1 \mathrm{mg} \sim 1 \mathrm{~g}$ & $\mathrm{E} 2$ & 1 sets \\
\hline $\begin{array}{c}\text { Standard } \\
\text { weights }\end{array}$ & $1 \mathrm{~g} \sim 6 \mathrm{~kg}$ & $\mathrm{E} 2$ & 1 sets \\
\hline
\end{tabular}

It can be seen from table 3 that the accuracy and repeatability of the development of the special traceability measuring tool for micro force value are $0.005 \%$ and $0.003 \%$, meeting the design and use requirements. 
Table 3 Test Results

\begin{tabular}{|c|c|c|c|}
\hline $\begin{array}{c}\text { Standard } \\
\text { value } \\
(\mathbf{g})\end{array}$ & $\begin{array}{c}\text { Test } \\
\text { value } \\
(\mathbf{g})\end{array}$ & $\begin{array}{c}\text { Error } \\
(\mathbf{\%})\end{array}$ & $\begin{array}{c}\text { Repeatability } \\
(\mathbf{\%})\end{array}$ \\
\hline 1.0000 & 1.0000 & $0.000 \%$ & $0.001 \%$ \\
\hline 2.0000 & 2.0001 & $0.005 \%$ & $0.000 \%$ \\
\hline 5.0000 & 5.0002 & $0.004 \%$ & $0.001 \%$ \\
\hline 10.0000 & 10.0000 & $0.000 \%$ & $0.001 \%$ \\
\hline 20.0000 & 20.0002 & $0.001 \%$ & $0.002 \%$ \\
\hline 50.0000 & 50.0000 & $0.000 \%$ & $0.000 \%$ \\
\hline 100.0000 & 100.0009 & $0.001 \%$ & $0.001 \%$ \\
\hline 200.0000 & 200.0012 & $0.001 \%$ & $0.000 \%$ \\
\hline 300.000 & 300.001 & $0.000 \%$ & $0.002 \%$ \\
\hline 500.000 & 500.000 & $0.000 \%$ & $0.000 \%$ \\
\hline 1000.000 & 1000.001 & $0.000 \%$ & $0.003 \%$ \\
\hline & & \multicolumn{2}{|c}{} \\
\hline
\end{tabular}

\section{SUMMARY}

This paper introduces the development of a special measuring tool for micro force traceability, which has a measuring force range of $0.009807 \mathrm{~N}$ $9.807 \mathrm{~N}$ ( $1 \mathrm{gf} \sim 1 \mathrm{kgf})$, and the accuracy is better than $\pm 0.1 \%$. It includes the selection of electromagnetic balance module, the design of hardware structure, the determination of measurement accuracy, and the compilation of software program. Finally, a special traceability measuring tool which integrates measurement, automatic data acquisition and data processing is realized, and the experimental verification results are given. At the same time, it has been well applied in our laboratory. The special inspection tool for trace of micro force provides a new method to solve the problem of micro force traceability of micro Vickers hardness tester and Knoop hardness tester. The next step is to develop a small force traceability tool with smaller geometry for most micro Vickers and Knoop hardness machines.

\section{REFERENCES}

[1] ISO/TC 164, ISO 6507: Metallic materials-Vickers hardness test, 2018.

[2] ASTM E92 Standard test methods for Vickers hardness and Knoop hardness of metallic materials, 2017.

[3] ASTM E384 Standard test method for microindentation hardness of materials, 2017

[4] JJG 148 Verification regulation of Vickers hardness reference blocks, 2006.

[5] JJG 151 Verification regulation of metallic Vickers hardness testers, 2006.

[6] Liu Maochun. Li Qi, C \# program development case class. New: tsinghua university press, Ch14, pp. 299-362, 2018. 